\title{
TRACTION ÉLECTRIQUE
}

\section{Par la Houille Blanche ou par la Houille Noire, l'électrification générale de nos chemins de fer s'impose.}

\author{
Par J. BOUDET, Ingénieur A. M., Membre de la Société des Ingénieurs civils de France \\ el de l' Associazione Elettrotecnica Italiana" (Section de Turin).
}

Sous de nombreux rapports ct pour de nombreuses gens, la guerre comporte tout à la fois, une foule de révélations el d'enseignements. Une des plus séricuses parmi les premières est, à coup sûr, l'état d'infériorité dans lequel se maintiennent nos chemins de fer, cristallisés autour de la traction à vapeur. Il est notoire que, malgré le magnifique effort qu'ils ont soutenu pendant loute la durée de la tourmente, ils sont tombés bien bas a certains des moments les plus sombres de notre histoire, et en particulier en 1917-1918, alors que sonnait pour nous l'heure décisive de ce duel gigantesque.

Pour être juste, il faut bien reconnaître qu'à cette époque ils avaient ì faire face, par une température rigoureuse, à un trafic démesurément accru par les circonstances ; il n'en est pas moins vrai que ces circonstances - même ont souligné de façon frappante linfériorité des transports à vapeur, - (et cela non seulement en France, mais partout ailleurs, - on pourrait prescue dire dans le monde entier), en particulier dans les services où ces derniers ont pu ètre mis en parallèle avec les transports électriques.

Il semble bien quill ne reste plus rien à dire pour établir la supériorité de ceux-ci, et que, depuis qu'on l'agite, la question a dû être épuisée.

Il est pourtant un côté qui nous paraît avoir été négligé dans la plupart des éludes qui l'ont abordé : nous voulons parler de la nécessité qu'il y a désormais, et ce pour des raisons à la fois techniques et économiques, à substituer à notre antique traction à vapeur, que nous étions trop habitués depuis un siècle, à considérer comme la caractéristique essentielle de toute idée de chemin de fer, lo nouveau mode de traction, plus moderne, plus puissant, el surtout plus économique, parce que micux adapté à lirrégularité et à l'intermittence des efforts à fournir dans toute exploitation ferroviaire, ceci quelle que soit la source à laquelle la traction électrique pourra demander son énergie.

On a lcllement répélé au grand public que l'électrification des chemins de fer était fonction de laménagement de nos forces hy drauliques avec lequel elle paraît - du moins pour le moment devoir marcher de pair, que le public - el avec lui eneore, certains milieux techniques - croient volonliers que les deux choses sont inséparables et que scule la houille blanche est susceptible de permettre l'électrification de nos voies ferrées. La présente áde a pour objet principal de démontrer qu'il n'en est rien et: que la houille noire, rationnellement consommée, peut et doit, au mème litre que la houille blanche, concourir à l'électrification générale envisagée.

N'ayant encore, en France, que de minuscules sections de lignes électrifiées (et dans des conditions bien différentes) sur lesquelles peu ou point de statistiques sont établies, nous nous réfèrerons, pour élayer ce qui va suivre, aux nombreuses statistiques américaines, récemment publićes au cours des discussions que la question a suscilées outre- $\Lambda$ llantique et, plus particulièrement encore, à celles du "Chicago Milwaukee and St-Paul-Railway ": à côté de 660 milles de grandes lignes, complètement électrifiées depris longtemps (1), cette Compagnie possède un vaste réseau exploité à la vapeur et ses statistiques comparatives entre les résultats obtenus avec les deux systèmes de traction sont, à cet égard, particulièrement intéressantes. Toutefois, il ne paraît pas inutile, auparavant, de résumer brièvement ici, — même si cela doit constituer, à certains points de vue, une répétition - les principaux avantages que la traction électrique, systématisée sur tous les réseaux, est susceptible d'apporter rapidement dans l'exploitation de nos chemins de fer.

\section{I. - Rappel des avantages à attendre de l'électrification générale.}

Aucun de ces avantages n'est négligeable ; tous représentent, par rapport à la traction à vapeur, des améliorations tangibles quand ce ne sont pas des progrès réels, parfois considérables ; néanmoins, il en est dont lintervention isolée, pour appréciable qu'elle soit, ne saurait évidemment justifier, à elle seule, une transformation aussi profonde du système de traction. Parmi ceux-là nous citerons :

La suppression de toutes souillures et incommodations pour les voyageurs, en même temps que de toutes fumées — suppression aussi intéressante pour les voyageurs (et aussi pour le personnel des trains) que pour les habitants des environs des gares, particulièrement dans les villes, dont la salubrité se trouve de ce fait améliorée.

La suppression complète des étincelles, et, avec elles, de tout danger d'incendie, permettant la revalorisation des propriétés voisines du chemin de fer ;

La suppression à peu près complète des installations et des dépenses relatives à l'approvisionnement en cau des chaudières.

La suppression des tenders, plaques tournantes, fosses à piquer, etc... ;

La grande rapidité de mise en marche, avec un effort de traction très élevé ; l'influence, presque négligeable, du profil des lignes et des conditions climatériques sur l'exploitation ;

La possibilité de très grands parcours sans aucun besoin de ravitaillement ni de révision ;

Enfin, l'économie de personnel : un seul agent suffisant à la conduite des automotrices; la diminution des risques d'accidents

(1) Y compris les 208 milles livrés au trafic électrique au printemps de 1921 (Nous rappelons que le mille anglais (terrestre) équivaut à $1,609 \mathrm{Km}$.) 
dûs tant aux emballements dans les descentes qu'à l'inobservation des signaux par le mécanicien : ce dernier, à peu près déchargé de tout travail ou effort manuel, pouvant se consacrer bien davantage à la surveillance de la roie; et une possibilité de variations maxima, dans de très bonnes limiles de rendement, de l'effort de traction et de la vitesse des convois:

A côlé de ces avanlages, d'ordre secondaire, malgré leur intérêt, - au moins poùr l'objet de cette discussion, - il en est dautres, dont la scule considération constilue, ainisi qu'on va l'établir, un facleur capilal, voire décisif, on faveur de la généralisation de ce systeme de traction. Au nombre de ces demiers avantages, nous citerons :

Une notable réduction dans les dépenses dexploitation, de par la réduction des poids morts (chaudières et tenders) - ainsi que dans celles d'entretien et surtoul de réparations : chaque automotrice étant capable de fournir, en moyenne, un parcours annuel de plus de 80.000 kilomètres, alors que les locomolives à vapeur n'en fournissent qu'exeptionnollement une cinquantaine, sans passer par l'atelier (1) ;

Une considérable possibilité d'augmentation du trafic avec un mouvement de convois à peine aceru, ct d'ajournement - souvent mème d'évitement - des dédoublements de voies el des élargissements de plateformes correspondants, si onéreux en pays de

(1) Notoire est l'endurance de certains de ces engins, parmi lesquels on peut citer tout spécialement les automotrices triphasces du Fréjus, qui accomplissent annuellement plus de 100.000 kilomètres dans des conditions constructives et altimétriques correspondant à une distance virtuelle d'au moins $250.000 \mathrm{kms}$. (Voir, à ce sujet, la discussion ouverte dans les colonnes de l'Elettrotecnica, au cours de l'anncé dernière, 1922).

Par ailleurs, les dépenses entretien-réparations sont particulièrement réduites, ainsi qu'il ressort du tableau ci-dessous, dont les statistiques, effectuées sur deux réseaux différents se recoupent sensiblement, si l'on tient compte de la différence de tonnage des engins èn sèrvice sur ces réseaux. (A noter, toutefoìs, que ces dépenses (qui ressortent à environ 3 cents par 100 tonnes d'automotrice-mille) sont relatives à des engins à courant continu et que les engins à courant alternatif dépensent environ le double, ce qui est encore peu, comparé aux locomotives à vapeur.

\section{TableaU I}

Dépeñsés éntretien-réparations des automotrices américaines

\begin{tabular}{|c|c|c|c|c|c|c|c|c|}
\hline \multirow[t]{2}{*}{$\begin{array}{c}\text { Eléments } \\
\text { des Statistiques }\end{array}$} & \multicolumn{5}{|c|}{$\begin{array}{l}\text { Réseau du } \\
\text { New-York Central }\end{array}$} & \multicolumn{3}{|c|}{$\begin{array}{c}\text { Réseau du } \\
\text { Chicago- } \\
\text { Milwaukee } \\
\text { and St-Paul Rly }\end{array}$} \\
\hline & 1914 & 1915 & 1916 & 1917 & 1918 & 1918 & 1917 & 1918 \\
\hline $\begin{array}{l}\text { Nombre d'automotri- } \\
\text { ces en service..... } \\
\text { Tonnage ünitaire }\end{array}$ & 62 & 63 & 63 & 73 & 73 & 20 & 44 & 45 \\
\hline $\begin{array}{l}\text { Dépenses par auto- } \\
\text { motrice-mille.... }\end{array}$ & 4,03 & 4,45 & 3,78 & 4,01 & 6,26 & 8,21 & 9,62 & 10,87 \\
\hline
\end{tabular}

Pàr ailleurs, et en corrélation étroite avec ces statistiques, ajoutons qu'aux termes du rapport de 1917 de M. E. Furneau, à l'" American Rly Engineering Ass. ", il résulte que les essais effectués tant sur les réseaux ci-dessus que sur le "Norfolk and Western" et sur le "Schenectady N. Y. " ont établi que l'effet d'impact des áutomotrices électriques sur le matériel roulant, très inférieur à cèlui des locomotives á vapeur, ne dépasse pas $30 \%$ tant que les châsisis sont de longueur inférieure ou égale à 8 metres: monlagnes comme aux abords des villes, dont, bien souvent, l'électrification, - malgré ses frais élevés de premicr établissement - constitue l'unique solution possible.

Particulièrement éloquents sont, à ce sujet, les résultals obtenus dès son électrificalion, sur la division des MonLagnes Rochenses du "Chicago-Milwaukee and St-Paul Rly », ou la traction électrique a permis d'assurer le mème trafic que la traction à vapeur,

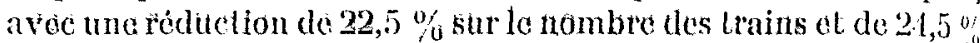
sur la durée des parcours, améliorant lẹs conditions générales de lexploitation au point que le trafic actuel, supérieur d'un ticrs à celui de celle époque, est assuré en moins de $80 \%$ du lemps que nen demandail, autrefois, la traction à vapeur pour un lomnage sensiblement moindre, ce qui représente une augmentation de plus de $50 \%$ dans la capacité de la ligne, ajournant sine die, et ad víam, sans doute -, son dédoublentent, envisagé bien longtemps avant l'ćlectrification, et justiftant plusicurs fois la dépense engagée pour cetle dernière (1);

En corrélation étroite avec celte demière et très importante considération, la suppression de tous les transports inutiles, en charbon el cn eau, nécessilés par la traction à vapeur et dont l'inportance, généralement trop peu soupçonnéc, vient d'etre mise en lumiere par une étude approfondie, faite sur lo " $\mathrm{B} \mathrm{et} O$. Fird" touchant les circonstances d'embouteillage de ses voies, sur la section comprise entre Grafton et Cumberland.

Il résulte, en effet, de celle étude trìs serrée, que le trafic du charbon de la Compagnie, transporté tant sur wagons que sur tenders, représentait plus de $11 \%$ du trafic total, en tonnes-milles, passant sur ces lignes, ou, cn aulres termes, --- pour employer une figure plus tangible - que un train sur 9 était régulièrement nécessaire pour assurer le transport du charbon brûlé par les locomotives ; alors qu'avec le programme d'électrification actuellement envisagé on compte non sculement assurer la liberté des trois voies congestionnées; mais encore les rendre capables, dans l'avenir, d'un tráfic accru d'au moins $80 \%$;

Enfin, la suppression - particulieremenl importante - de toute consommation à l'arrêt, en cutre d'une considérable économie de combustible pendant la marche, considérations majeures dont le développement fait l'objet de deux paragraphes du chapilte suivant.

\section{II. - Une centrale thermique fournissant à la traction élec- trique est beaucoup plus économique que les locomotives de la traction à vapeur.}

C'est aujourd'hui un lieu commun de dire que la locomotive électrìque est, par essence, anti-économique, mais on se rend mal compte, généralement, du véritable gaspillage de combustible inhérent à l'emploi de la vapeur pour la traction ferroviaire La statistique ci-dessous, empruntée au rapport de M. Arnstrong au Comité d'électrification de la Compagnie Générale élcetrique de Schenectady (2) le met en évidence, car il en ressort clairement que, pendant la seule année 1918, un quart de la totalité des combustibles fossiles extrails aux Etats-Unis a été consommó par los dien mins de fer à vápeur.

(1) Sur la division électriliéc du *Ch. M. and St P. Rly" 42 automotrices électriques ont remplacé avec un capacitó dêt transport et de réscrve beaucoup plus grandes, 112 locomotives vapeur.

(2) Electric Rly: „Jat, T. 55, N. 8 (Déclarations de M. Armstrong, Président de l'Electrification Commiltee of the General Electric Company $"$. 
Tablaka II

Production do combustibles ol consammalian forroviaive au U. S, A., en 1918

Production totale de charbons, loules calégories, Tonnes.............

Charbons consommés par les eliemins de

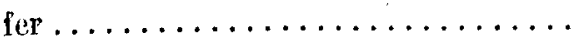

Combustibles liquides extraits dans le pays : Barrils...............

Combustibles liquides consommés jar les chemins de fer : Barpils.........

Equivalence, à raison de 3,5 bls par tonne: Tonnes................ Consommation tolale des chemins de fer. Tonnes.................

Soit, en pourcentage du total. . ., ......
$678.211 .000 *$

$163.000 .000 *$

355.927 .000

45.700 .000

$13.600 .000^{*}$

$176.000 .000 *$

$24 \%$
PINTES EN STATION, INHERENTES A LA TRACTION A VAPECR

Par ąilleurs, dès 1910, des expériences serrées avaient été faites sur la section des Montagnes Rocheuses du Chicago Milwaukee and St-Paul Rly", pour déterminer la relation existant entre le trạvail effechif, fourni par les locomolives à vapeưr cn servịce sur cette scelion et leurs différenles consommations de combuslibles.

Elles avaient permis d"étaplir qu"en travail effectif, sur cette ligne accidentée, les conssommations s'élevaient, en moyenne, toutes pertes réduites, à :

39,86 Ih. (soit 14,5 ligs) deeat par CVh ;

5.06 Ib. d'eau par Ib. de charbon :

- (soit 5,5 kgs par leg de charbon ;

at 7,80 Ib. de elariton (soil $2.850 \mathrm{kgs}$ ) par CVh ;

avec, comme pertes sèches, pour le parcours entier :

Ralculissencint des feux en rolonde $150 \mathrm{lb}$. de chạbond à lineure

$$
54.500 \mathrm{kgs})
$$

Nelloyage des feux au depart... $800 \mathrm{lb} .-290 \mathrm{kgs})$.

Parcours en descentes......... $950 \mathrm{lb}$ - $345 \mathrm{kgs}$ )

Arrêts pour croisemenls, garages,

$$
\text { elc............... } 500 \mathrm{lb} .-182 \mathrm{kgs})
$$

En ajoulant ces pertes en station anx $2.850 \mathrm{kgs}$ par CWh indiqués ci-dessus, et en divisant la consommation totale de charbon ent 24 heures par le trạvail effectif foumi dans le mẹme temps, on tronve que ce travail a été oblenu au prix d'une consommation unitaire de $3,700 \mathrm{kgs}$ de charbon par CVh effectif, fournị aux jantes.

La concịsion qui se dégage des résultats fournis par ces expériences esl que les pertes en stalion augmentent de $30 \%$ la consommatiou de charbon pendant la période de travail utilie, ou, cul aulres lermes, que le tiers du combustible brûlé par les chemins de fer est complètement perdu.

Comme complément aux essais précilés, une statistique de 30 jours conséculifs fut tenuc, de la consommation totale du charbon pour la section entięre des Montagnes Rocheuses et dụ trafic lotal des machines-tenders el trains, ce dernier traduit en CVh,

Cetle statistigue permit de fixer a $10,53 \mathrm{lb} .(3,820 \mathrm{kgs})$ le poids du combustible brûlé, par CVh aux jantes.

$\left({ }^{*}\right)$ Dans ce tableau, comme daus les suivants, les nombres expriment des tonnes anglaises, qui n'ont point été converties en tonnes niétriques, en raison du peu de différence existant entre cos deux unités ( + 16 k kgs).
Les deux valeurs ci-dessus étaient basées sur les constantes ci-après : $6 \mathrm{lb},(2,200 \mathrm{kgs})$ par tonne-train d $\beta$ résistance à toutes les vitesses, et $0,7 \mathrm{lb} .(0,250 \mathrm{kgs})$ par tonnne et par degré de courbe, déterminées par les essais dynamométriques aux vagons et considérées commẹ synthétisant le trafic général de la lígne.

En rapportant la moyenne des yaleurs du charbon brâlé pendant les trajets d'épreuves et la statistique des 30 jours, par $\mathrm{CVh}$, aux constantes électriques hạbituelles, on obtient les valeurs consignẹécs au tạbleạu ci-après :

\section{TABLEAU III}

Consommations unitaires de charbon avec la traction à vapeur :

Charbon, par CVh, aux jantes des roues motrices. .......... b $10,27 \quad(3,730 \mathrm{kgs})$

Charbon, par KWh, aux jantes des roues motrices ......... $13,75 \quad(5,000, \mathrm{kgg})$

Consommation unitaire de charbon correspondant, sur la base d'un rendement de $55 \%$, à l'utilisation

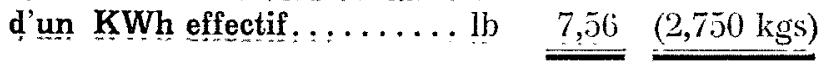

C'est ce chif̣re, souligné, de $7,56 \mathrm{lb} .(2,750 \mathrm{kgs})$ de charbon brûlé sụr lẹs locomotives à yapeur pour obtenir, en trạfic ṇormal, un effort correspondant à $1 \mathrm{KWh}$ fourni par une centrale électrique, qui eșt d'un intérêt capital pour cette discussion.

D'autre part, une comparaison entre la consommation du charbon de la "Butte Anaconda and Pacific Rly", avant et après son électrification, fait ressortir un poids de $7,17 \mathrm{lb} .(3,100 \mathrm{kgs})$ brûlées précédemment sur les locomotives à vapeur, alors que, pour assurer désormais le même service, il suffit d'un KWh fourni par les sous-stations. Ces chiffres, comme on le voit, recoupent de saisissante façon ceux ci-dessus, établis par un procédé entièrement différent.

\section{ECONOMES DE CONBUSTIBLE \\ RÉALITSABLES PAR L'ÉLECTRIFICATION GÉNÉRALE}

Si l'on tient compte de ce que le charbon utilisé lors de l'établissement de ces stalistiques présentait un pouyoir calorique quelque peu faible, on pent, à coup sûr, affirmer que les chamins de fer à vapeur consomment le charbon au taux véritablement effarant de $12,75 \mathrm{lb} .(4,600 \mathrm{kgs})$ par $\mathrm{KWh}$ de travail utile mesuré aux jantes, ou $7 \mathrm{lb}$. (2,540 kgs) par KWh mesuré au wattmètre d'une centrale, et comprenant - pour la rigueur de la comparaison - les pertes en ligne et en conversion inhérentes à l'emploi de la traction électrique. Or, il est notoire que le KWh peut-être produit, en centrale, par la combustion d'un poids de charbon n'excédant jamais $1,500 \mathrm{kgs}$ dạns les usines anciennes, même démodées, et descendant jusqu'à $0,800 \mathrm{kgs}$ dans les nouvelles centrales pourvues des derniers perfectionnements (Genevilliers)...

Il est, dès lors, facile d'évaluer l'économie considérable qui résulterait d'une électrification générale : êle ressort des tableaux ci-après, élablis par M. Armstrong pour l'ensemble des chemins de fer des Etats-Unis (1).

(1) Toutes ces données sont rapportées à l'énergịe distriluuée, sous tension de 100.000 volts, par la "Montana Power $C^{\circ}$, compte tenu des récupérations par freinage sur les pentes, et dont le total atteint $14 \%$ de l'ensemble. Or, en raison du profil très accidenté de la partie électrifiée du Ch. M. and St-Paul "sur laquelle ont porté les statistiques, cette opération étant particulièrement rémunératrice, la consommation étalonnée, de 33,3 watts-heure par tonne-mille (soit 20 watts=heure par TK.), pour l'ensemble du trafic voyageurs: marchandises, doit certainement être augmentée pour la mettre en harmonie avec celle constatée sur des profils plųs réguliẹs, et 
TABLEAU IV

Statistique reconstitutive du charbon nécessaire aux chemins de fer des U. S. A., en 1918

Trafic assuré, en tonnes-milles. TM soit, en tonnes-kilométriques $\mathrm{Tk}$

1.215 .400 .000 .000

Watts-heure, par tonne-mille.....

1.987.200.000.000 soit, par tonne kilométrique....

40

Consommation totale pour l'ensemble du trafic...... KWh

Charbon nécessaire, à raison de $7 \mathrm{lb}$ (soit $2.540 \mathrm{kgs}$ ) par KWh.T.

48.700 .000 .000

170.000 .000
Comme on le voit, les résultats de ce tableau IV recoupent sensiblement les statistiques données par le tableau II ci-dessus.

ce n'est point la surestimer que de la considérer comme égale, en chiffre rond, à $40 \mathrm{Wh}$ (soit 24,5 Wh par TK).

Les expériences effectuées sur le parcours de Harlowtown à Three-Forks, et dont les résultats sont consignés dans les graphiques ci-contre, sont, à cet égard, particulièrement suggestifs.

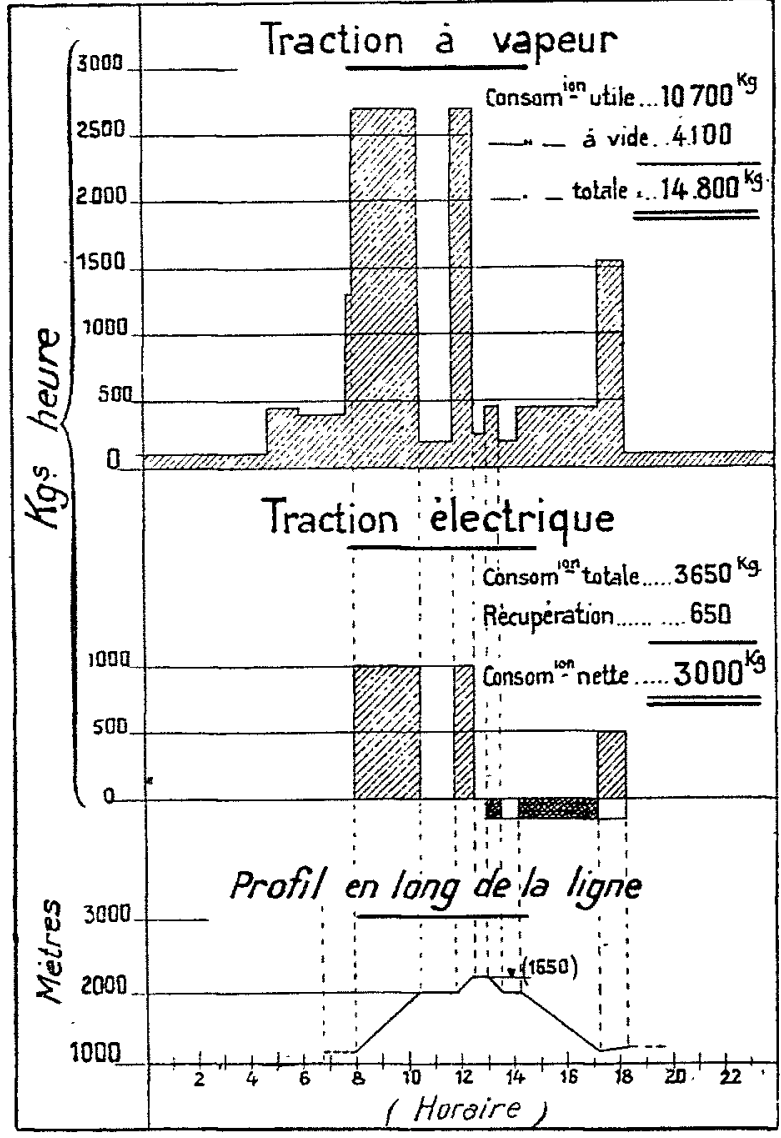

Fig. 1

Graphiques comparatifs des consommations etalonnées avec les deux systèmes de traction, sur la section Ifarlowtown-Three fillds de la ligne des Montagnes Pocheuses (Ch. M. and St Paul Rly).

Sur ce parcours, les locomotives à vapeur remorquaient des trains de 26 voitures, pesant 900 tonnes, et les automotrices électriques, des convois de 2.800 tonnes comportant jusqu'à 64 wagons, à des vitesses légèrement supérieures. Les diagrammes ci-dessus indiquent les consommations respectives pour chaque système de traction.

Sur les locomotives à vapeur, on brûlait du charbon à 6.600 calories environ par kilog. strictement pesé aux différentes stations d'approvisionnement, et, par ailleurs, la consommation des locos électriques qui utilisaient de l'énergie d'origine hydraulique était mesurée au moyen des appareils habituels placés sur chacue machine, dont les indications étaient ensuite rapportées à la consommation de la centrale, calculée sur la base d'une utilisation moyenno de $67 \%$, reconnue comme normale, et le chiffre de consommation transformé ensuite en poids de combustible, à raison de $1,130 \mathrm{kgs} \mathrm{de}$ charbon par KWh, représentant une bonne moyenne pour les centrales quelque peu modernes.

\section{TABLEAU V}

Economies de charbon possibles avec l'électrification (Chemins de fer des U. S. A.)

Total du trafic général ci-dessus : TM (soit, en TK)............... 1.215 .400 .000 .000

1.987 .200 .000 .000

Réduction dûe à l'électrification: TM. 146.000 .000 .000 (soit, en TK)............... 238.700 .000 .000

Trafic à vapeur, en TM...... TM (soit, en TK).............. 1.069 .400 .000 .000 1.748 .500 .000 .000

Consommation d'énergie (à 40 Wh par $T M) \ldots \ldots \ldots \ldots$
(ou 24,5 par TK) $\ldots \ldots$; KWh 42.776 .000 .000 Charbon nécessaire à cette production d'énergie, sur la base de 2,5 lb (0 kg,900) par KWh.... Tonnes 53.500 .000

Consommation totale de charbon sur les ch.de fer,étalonnée pourl'année 1918 (Tableau I)..... Tomes

176.000 .000

Economies réalisables par l'électrification $\ldots \ldots \ldots \ldots \ldots \ldots \ldots$

122.500.000

soit, en chiffre rond, $70 \%$ de la consommation totale.

La déduction qui se dégage du rapprochement de ce dernier tableau avec ceux qui précèdent, ct qui est soulignée au bas du tableau V, est qu'en cette année 1918, sur laquelle portent les statistiques de M. Armstrong, l'électrification générale des réseaux eût permis d'économiser plus des $2 / 3$ de la consommalion totale du pays, représentant en quantité $150 \%$ des exportations anglaises d'avant-guerre, et près de deux fois et demi l'ensemble de la consommation française actuelle, toutes industries réunies.

Par application de ce résultat à nos propres chemins de fer français, on trouve que, sur les 8.300 .000 tonnes de charbon consommées actuellement par leurs locomotives, plus de $\mathbf{5 . 5 0 0 . 0 0 0}$ tonnes, représentant, aux cours astuels, une valeur de près d'un demi-milliard de francs - pourraient être économisées sans aucun changement dans la nature de nos combustibles consommés actuellement, mais seulement dans le mode de consommation, et par la substitution, généralisée, de la traction électrique à l'antique trątion à vapeur.

\section{Crise mondile de Combustibles et Programmes d'Electrification}

Si l'on se reporte à l'annéc critique sur laquelle ont porté les stalistiques qui précèdent, - lout comme on peut le fair, ćgalement, à ses suivantes, qui n'en diffèrent pas sensiblemcnt, il faut bien reconnaitre que la situation mondiale, et, en particulier celle de la France (qui ne s'est guère améliorée depuils cette époque) contrastait, et contraste encore étrangement aver le gaspillage qui ressort des dites stalistiques, et à côté duquart il nous faut, hélas ! faire état, - en outre d'une formidable dette de guerre supportée par une population amoindrie d'un douzième - des dévastations de nos plus industricuses régions du Nord et de l'Est et de la destruction systémalique de leurs houillères, dont la production est lombéc, de 40.000.000 tonnes en 1913 à 20 millions seulement on 1920, obligeant à uso importation de 25.500.000 tonnes, qui dépasse sensiblementl celle d'avant-guerre, alors que la consommation a dimisutum

Or, il est avéré que la restauration complete des mines die truiles demandera encore six à huil années d'elforls, pendant lesquelles ces importations ne peuvent que s'accroître au. fur ell à mesure que notre consommation se rapprochera du clififfoe d'avant-guerre (63.000.000 de tonnes), car te secours apponth 
par le bassin de la Sarre (moins de 6 millions de tonnes) est un appoint bien minime, sinon négligeable (1).

La siluation demande non des pallialifs, mais des remèdes énergiques. On s'en est pleinement rendu comple en haut licu, et, depuis la fin de la guerre, nos chemins de fer ont un programme d électrification qui commence - quoique bien lentement - à entrer dans la voie des réalisalions. Mais ce programme, presque limide eu égard aux nécessités de l'heure, ne comporte encore qu'une partie vraiment trop restreinte de leurs grandes lignes ( $1 / 5^{\circ}$ environ) (2) choisies de préférence parmi celles des régions monlagneuses, riches en houille blanche, mais généralement assez pauvres en trafic ferroviaire ; ct ce choix même est bien do nalure à accréditer l'assertion que nous voudrions contribuer ¿̀ détruire, à savoir que l'idée d'électrification est inséparable de celle de "houille blanche ».

Par ailleurs, il faut bien reconnaîlre aussi que, sur la base de 100 kilomètres par an (qui n'onl point encore élé atteints), l'électrification de celle seule partie des réseaux montagneux demandera plus de 20 ans, et qu'elle ne constitue, par cela même, qu'un remède partiel autant qu'à échéance lointaine. Cela suffit pour conclure qu'il est insuffisant et que, dans les régions dépourvues de houille blanche - qui, pour cette raison, ont été exclues dı programme d'électrification élaboré - la houille noire peut et doit être appelé à la remplacer, contrairement à ce qu'on pense trop généralement, pour assurer une production rationnelle d'energie, distribuée de la mème façon que celle d'origine hydraulique, et qui sera encore, comme on vient de le voir, beaucoup plus économique que sa consommalion directe sur les locomolives à vapeur, même des modèles les plus récents et les plus perfeclionnés, dont le rendement maximum atteint péniblement $10 \%$ de la puissance; qu'il est possible d'obtenir avec leur consommation de combustible (1).

(1) D'une façon générale, toutes les nations européennes de J'Ouest touchées par la guerre, sont logées à la même enseigne et se rendent pleinement compte, aujourd'hui, du danger qu'il y a pour la vie nationale, à demeurer tributaire de l'étranger en matière de combustibles.

Complètement dépourvue de combustibles fossiles, et obligée d'importer chaque année, ses 10 millions de tonnes de charbon, l'Italie nous a devancés. sur la voie de l'électrification générale. Quoique en situation moins précaire, parce que plus rapprochée des centres de production et d'exportation allemands, la Suisse poursuit activement la réalisation d'un très vaste programme. La Belgique elle-même, dont les mines n'ont pas souffert de l'invasion, mais qui n'en sent pas moins impérieusement le besoin d'éconamiser ses réserves, a, elle aussi, son programme d'électrification. Enfin, il n'est pas jusqu'à la vieille Angleterre qui, bien que vivant de ses exportations de combustibles, ne songe, pour ses propres besoins, à une utilisation meilleure et plus rationnelle que celle fournie par les machines à vapeur actuelles, et il ne paraît point téméraire d'envisager qu'avant 10 ans elle ait réalisé beaucoup plus que ses alliées.

(2) En chiffres ronds, environ $8.200 \mathrm{kms}$, répartis comme suit : Réseau du Midi, environ.......... $3.000 \mathrm{kms}$

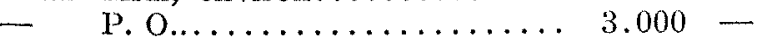

$$
\begin{aligned}
& \text { - P. L. M................. 2.200 - } \\
& \text { Total égal............ } 8.200 \mathrm{kms}
\end{aligned}
$$

(1) Les locomotives actuellement en service sur nos chemins de fer - au nombre de 13.000 - consomment anmuellement - comme on vient de la voir - 8.300 .000 tomnes de charbon, soit à peu près la consommation de l'agglomération parisienne (toutes industries réunies), représentant, environ (a $1 \mathrm{Kg}$ par CVh effectif), $800.000 \mathrm{CV}$ eflectirs en marche réguliere et continue pendant toute la durée de l'année.

C'est celle puissance qu'il suffirait de fournir aux machines si l'intensité du trafic était elle-même régulière et constante. Or, il est si loin d'en être ainsi, que la puissance totale réelle des locos sous pression en permanence dépasse, pour l'ensemble de nos réseaux, 10.000 .000 de CV.

\section{III. - Conditions de réalisation rationnelle de l'électrification ferrovialre générale.}

\section{Besoins eN ENergie}

De ce qui précède, on peut déduire approximalivement une estimation de l'énergie qui serait nécessaire pour une électrification générale de nos réseaux ferrés; elle n'est pas formidable.

En effet, si l'on se reporte aux statistiques qui précèdent, on peut, sur la base de $40 \mathrm{Wh}$ par TM (soit 24,5 Wh par TK) estimer la consommation annuelle, probable, des automotrices, à :

KWh, environ..........

2.200 .000 .000

correspondant - avec un facteur de charge de

$100 \%$ - à une puissance instantanée, aux

jantes, de........................

soit, en centrale, avec un facteur de $50 \%$, KWS.

en chiffres ronds, par excès (1).

Ce chiffre des besoins ferroviaires représente, à-peu près, la puissance totale des usines hydrauliques, ou, si l'on préfère, environ la moitié de celle des us nes thermiques équipées à ce jour en France. Sans tenir compte de celles projetées (2), - dont la construction ne sera un fait accompli que dans un avenir indéterm:né et, à coup sûr, lointain, — si l'on considère que, avec les usines actuellement en cours de construction, et dont la puissance est prévue pour seulement $580.000 \mathrm{KWS}$ (dont 220 d'origine thermique et 360 d'origine hydraulique), la France ne disposera, dans un avenir relativement prochain estimé à une quinzaine d'années, que de $2.250 .000 \mathrm{KVS}$, alors que ses besoins, d'ici cette époque, semblent devoir atteindre sinon dépasser $3.500 .000 \mathrm{KWS}(3)$, il en ressort clairement :

D'une part, que la seule "Houille Blanche " ne saurait suffire pour assurer - tout au moins rapidement - à la fois les besoins agricoles et industriels du pays et ceux, plus impérieux encore, de ses chemins de fer.

D'autre part, que ces derniers besoins, contrairement à ce qu'on imagine généralement, sont d'ordre secondaire, comparés aux premiers.

Or, il est encore possible d'envisager une notable réduction de ces besoins ferroviaires, par l'amélioration du facteur de charge que nous avons estimé à $50 \%$ dans ce qui précède, et qui paraît susceptible d'une augmentation aussi sensible qu'elle est désirable, si l'on envisage - ainsi qu'il est rationnel - le développement des réseaux interconnectés distribuant à la fois aux chemins de fer et à l'industrie par les mêmes lignes. A cet égard, du reste, il est intéressant de souligner les résultats déjà obtenus dans cette voie par le "Ch. M. aud St-Paul Rly" qui, avec des charges de pointes formidables, a néanmoins réussi à porter à près de $60 \%$ - ainsi qu'il ressort du tableau ci-après - le facteur de charge de l'ensemble de sa zone électrifiée, par la seule installation de limiteurs indicateurs de puissance, avant laquelle installation, ce facteur s'établissait péniblement aux environs de $40 \%$ (4).

(1) Dans son intéressante relation, dont les conclusions étayent du reste, la dissertation ci-dessus, M. Armstrong évalue à seulement 10.000.000 de KWS l'énergie nécessaire à une électrification générale des chemins de fer des Etats-Unis, soit seulement la moitić de la puissance équipée dans le pays, tant hydraulique que thermique, au cours des 30 dernières années. On se rend facilement compte que ces nécessités ne sont pas formidables.

(2) Pour environ 4.000.000 de KWS (dont 3.500.000 d'origine hydraulique et 500.000 d'origine thermique).

(3) Chiffres cités par M. Le Verrier, dans son remarquable rapport à la "Commission interministérielle pour la diffusion de l'énergie électrique dans les campagnes ».

(4) Ces installations feront l'objet d'une prochaine relation, a paraitre dans ces colonnes. 
Tableau 11

Facleurs de charge, en fon tion de; charges d: pointe, sur le "Chicago-Milwaukee" (4)

\begin{tabular}{|r|c|c|}
\hline Date des statistiques & Dụrée dos pointes & $\begin{array}{c}\text { Factẹurs de charge } \\
\text { correspondants }\end{array}$ \\
\hline 1919 Avpil ...... & 6,4 & 50,3 \\
Mai ..... & 4,6 & 56,1 \\
Juin ..... & 1,6 & 55,5 \\
Juillet ... & 0,7 & 55,6 \\
Août ...... & 4,1 & 54,7 \\
Septembre.: & 9,5 & 58,8 \\
\hline
\end{tabular}

Il est facile de se rendre compte qu'au point de vue commorcial un facteur de charge égal ou supérieur à $60 \%$ rend le ohemin de for électrique suffisamment intéressant pour permettre aux compagnnies de distribution de lui consentir des tarifs spéciaux. A forliori, lorsque pareille fourniture ferroviaire est comb.née, sur les réseaux, avec celles d'éclairage et d'énergie industrielle, le facteur de charge de l'ensemble peut dépasser $70 \%$, et il devient évident quo la distribution se présente dans des conditions de régularité très convenables pour enwisager la preduction permanonte qu'il est rationnel de demander à de grandes unsines modernes, connectées sur l'ensemble d'un réseau, quels que soient, du reste, les moyens de production de l'énergie (2).

\section{Production hydraulique et production therMilue} CONJUGUÉES

A l'heure actuelle, ayoc un fagteur de charge de $70 \%$ correspondant à une utilisation continue de 6.000 heures par an, en admettant pour pix de rexient du KWS disponible à l'usine, 2,500 à 3.000 francs - chịffres ạctuels - et 14 à $15 \%$ de oharges annuelles grevant l'exploitation, le prix de revient du KWh d’orrigine hydraulique s'établit aqux envirans de $0,0.7$ à 0,08 fr.

(4) Les chiffres de ce tableau représentant non des maxima mais lẹs moyennes d'obscrvations serrées faites, à la traversée dos Montagnes Rocheuses, pendant un semestre de trafic intensif, sur les 220 milles de lignes électrifiées alimentées par 7 sous-stations à contrôle şynchronisé.

Il semble bien que le taux de $60 \%$ soit encore susceptible d'augmentation sur les lignes à profil moịns accidentété,

(2) Un exemple typique de la nécessițé qu'il y a à améliarer les conditions générales de distribution, exemple emprunté au pays voisin dont il sera parlé plus loin, nous est fourni par l'alimentation du Chemin de fer des Alpes Bernoises, dont les caractéristiques sont résumées ci-après ;

Consemmation mentuguelle moyenne, à raison de

17 heures effectives par jour............ KWh

Moyenne de 6 pointes de 15 minutes........ IKWS

Facteur de charge, sur la base de 24 heures... (\%)

540.180

3.489

Semblables fluctuations dans la charge a demander au rósea

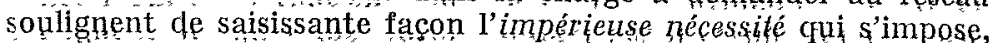
de la combiner avec d"autres charges, en particulier industrielles, de manière à maintenir le facteur de charge de l'ensemble du réseau aussi constant que possible et dans des conditions de travail convenables, ainsi qu'il est exposé ci-dessus.

Toutefois, en ce qui concerne les charges de pointes, le rapprochement de ces données avec celles du " Ch, M. and St-Paul Rly », dont nous ayons fait état, est difficile ; le facteur de charge de $60 \%$ qui caractérise l'utilisisation de l'énergie sur ce réseau, représentant le rapport de la moyenne à la pointe momentanée, alors que la charge de pointe du Lotschberg représente la moyenne de 6 pointes de 15 minutes, avec pointes momentanées beaucoup plus fortes. ahiffes qua l'on peul considoror commo de faibles moyonnas, sinon même comme des min ma.

Par contre, le $\mathrm{K}$ Wh d'origine thermique, produit dans de grandes centrales modenes (1), avee du charbon à 80 franes la tonne ot une consommation de $0,800 \mathrm{Kg}$ par KWh, cô̂le, en moyenue anviron 13 centimes (dont une moitié représente le seul combustiblo et l'autre les charges de toute nature).

$\mathrm{S}$, d'ici une quinzaine d'ạnées - marge d'avenir envisagée plus haut - le prix du charbon descend aux environs de $10 \mathrm{fr}$. la tomne (ce qui ne paraît pạs impossible), le prix de revient du KW Wh d'origine thermique pourra descendre au-dessous de $0,10 \mathrm{fr}$. et, devenant, dès lors, aussi intéressant que son concurrent hy. draulique, l'établissement do grandes centrạles thermịques sẹ présentera, pour les régions dépourvues de hoụille blanche mais pourvues de charbon ou le pouvant demander facilement à l'exportation directe (régions minières et littorales (2), dans des conditions particulièrement intèressantes et, en tout cas, sulfisamment avanlageuses (en particulier sur le cạreau des mines, oụ l'on peut brûler des combustibles de qualité médiocre, done peu marchands) pour concurrencer très largement, compte tenu des pertes, le prix du transport de l'énergie d'origine hydraulique amenée depuis ses régions de produclion, soit d'une distance susceptible de dépasser 500 kilomètres, distance à laquelle cọlle énergie a doublé de prix (3).

Toutefois, il semble bien logique d'apmettre qu'une pareille baisse dụ charbon ne se produira point sans une baisse corróla; tive des autres matières de consommation et dues matériaux de construction, peut-être mème d'une légère réduction des saslaires; et alors, tout à la fois, le prix de construction des usines hydrauliques aussi bien que celui des lignes de transport, el avec eux les charges annuelles des aménagements, subiraient des réductions correspontlantes. L'énergie d'origine hydraulique semble donc hịen appelée à concurrencer toujours avanlageusement collo d'origine thermique, au moins dans ses régions de pro. duction et leurs voisines immédiates; mais, comme on vient de le voir, dès qu'il s'agira de la transporter à des distances excédant 2 à 300 kilomètres, rendu aux pain lique reviendra à un prix au moins égal à son conourrent thermique.

Les deux ayant des rayons d'aclion elhnographịumement bien déţerminés, ne sauraient done se oncurrencer ; mais peuvent et doivont ôtre conjugués rationnollement du reste pour lo plus

(1) Le prix de rovient du KWS de puissance nopmalo clisponiblo, installé dans ees super-contrales, s'ótablit généralement aux environs de 800 francs, chiffe gui no sera pas meme atteint a Gennevilliprs, lossque l'éguipement sera complété.

(2) En attendant, pour ces dernières, l'industrialisation de la "Houlle bleue ", encore dans les langes...

(3) On compte, en effet, que l'énergie du Rhône, transportée à Paris, - soit sur 500 kilomètres environ, avec sculement 2 postes de transformation - reviendrait à $0 \mathrm{fr}$. 11 dans la capitale, si on la suppose à 0,06 sur lignes de départ. Envisagé dans de semblables condítions, c'est-à-dire avec deux postes, le transpont de l'énorgie de la Dordogne jusqu'à Nantes, sur 350 kilomètres, grèvora lo prix à l'usine de $0,04 \mathrm{fr}$.

Et l'on peut déduire, grossièrement, du rapprochement de ces deux cas concrets, que les transports à T. H. T. envişagés dans los conditions les plus favorables, grevent néanmoins le prix de revient du KWh d'environ $0,0.1 \mathrm{fr}$. par $100 \mathrm{Kms}$ de ligne, et que lorsfue le charbon sera descendu à $40 \mathrm{fr}$. la tonne, l'énergie d'origine thor mique conşommée dans są région da produçtion sęra plus économique que celle d'origine hydrauliquo dès que celte derniòe dewa être transportéc à plus de 4 à 500 kilomètres. Dòs lors, l'apération peut demeurer avantageuse, meme en brûlant du charbon dẹ bophe qualité. 
grand profit de la collectivité, dont les ressources seront ansı utilisées au maximum (1).

\section{STANDARdisation dE LA FRÉQUeNCE}

Reprenant la question de l'interconnexion entre centrales productrices d'énergie, quelle qu'en soil la nature, hydraulique ou thermique, nous croyons devoir ajouter quelques mots en ce qui concerne la fréquence, dont la standardisation s'impose si l'on envisage dans un avenir prochain la constitution d'un ou de plusieurs vastes réseaux de distribution couvrant la totalité du pays et fournissant à la fois aux chemins de fer, à l'industrie et à l'agriculture.

I ce sujet, il convient de noter la tendance actuelle, d'ordre général, voire mondial, qui se précise en faveur de l'adoption d'une frécquence unique, supérieure à loutes celles en usage. En Amérique, les stalistiques, tant publiques que privées (et parmi ces dernières, celles des ventes de la " G. E. C. \#) attestent qu'au cours des dix dernières années, la fréquence de 60 périodes a remplacé, en de très nombreuses stations, de plus basses fréquences, - en parliculier celles de 25 périodes à peu près intégralementel qu'elle tend, de plus en plus, à devenir la fréquence-type (ou standard), sans gèner en aucune manière le développement de l'électrification ferroviaire. En Europe, par contre, bien que la lendance vers l'accroissement soit tout aussi caractérisée, et quoique la situation ne soit pas encore aussi nette qu'en Amérique, on paraît toutefois vouloir s'en tenir à 50 périodes comme slandard.

Quel que soit le type auquel on s'arrête, il y a grand intérêt à en hâter la syslématisation.

Un exemple typique des graves inconvénients que présente, à cet égard, la manque d'uniformité, nous est fourni par la Suisse, payss où l'électrification est déjà très développée, mais se heurte journellement à des conflits de fréquences et de distribution qui deviement de jour en jour plus désagréables - quand ce n'est graves - et qu'éviterait complètement l'interconnexion (1), laquelle permettrait, en outre, à ce petit pays - au besoin, par une liaison avec ses voisins, ainsi qu'il vient d'être fait récemment, et qu'il sera prochainement fait, entre la Suède et le Danemark, une meilleure répartition des charges pour l'ensemble des besoins de la nation, et, partant, une utilisation beaucoup plus rationnelle de ses ressources nationales (2).

(1) C'est ce que traduit en chiffres le rapport, déjà cité, de M. Le Verrier, lorsqu'il dit " qu'on peut espérer, dans un avenir prochain " répartir sur tout le territoire, en des postes à 3.000-15.000 volts, "l'énergie électrique, à des prix pouvant varier, selon les régions et " les distances de transport, entre 6 et 18 centimes pour l'énergie : d'origine hydraulique, 13 et 18 centimes pour celle d'origine "thermicue "... (0,18 constitue bien, dans l'esprit de l'honorable rapporteur la limite à partir de lacuelle la première cesse d'être plus économique que la seconde, compte lenu des transports à très grandes distances).

(1) Particulièrement éloquent, à ce sujet, est le cas du chemin de fer des Alpes Bernoises (Berne-Lotschberg-Simplon), qui est alimenté à 15 périodes, alors que, dans la mëme centrale, des groupes à 42 périodes fournissent à l'industrie locale, et que toute la région environnante est desservic par un réseau à 50 périodes, nullement relié anx précédents... situation compliquée autant qu'irrationnelle pour tous les intéressés : compagnies, industries et particuliers, qui paient fort cher le maintien de ces trois fréquences, là où une seule suffirail à tous les besoins.

(2) Il semble bien, dans cet ordre d'idces, que la Suisse aurait

\section{IV. - Conclusion.}

En définitive, il résulte de ce qui précède :

Qu'en raison de l'acuité de la crise de combustibles qui étremt le monde entier, et du gaspillage de ces combustibles auquel nous assistons, avec la traction à vapeur, le remplacement de ce mode de traction, aujourd'hui suranné, s'impose d'urgence, et que l'électrification générale qui en doit marquer la disparition ne doit pas s'entendre seulement comme fonction du développement de l'utilisation de nos ressources hydrauliques, mais bien - et mieux encore - comme une des cellules du futur organisme que devra constituer, dans un avenir prochain, l'électrification générale du pays, bien comprise et réalisée rationnellement au moyen de centrales hydrauliques et thermiques interconnectées, alimentant un grand réseau national de distribution (Chambre des Députés, Délibération du 2 septembre 1919), dans le cadre de laquelle électrification générale rentrera logiquement l'électrification ferroviaire dont il vient d'être question, et dont la réalisation, avec la houille noire s'impose, au même titre qu'avec la houille blanche, puisque susceptible, ainsi qu'il vient d'être dit, de réduire de plus d'un quart nos importations de charbon actuelles.

Maintenant, pour bien mettre les choses au point, il ne paraît pas superflu d'ajouter que la dissertation qui précède n'envisage toutefois - comme électrification générale ferroviaire - que celle des lignes à grand trafic et à tracé tourmenté dont les résultats d'exploitation permettent d'envisager un amortissement relativement rapide des dépenses d'électrification.

Pour les autres lignes., dont la plupart sont d'une exploitation déficitaire - qu'elles soient, du reste, d'intérêt général ou d'intérêt local - la substitution de la traction électrique à la traction à vapeur ne saurait s'imposer (au moins avant très longtemps), en raison des dépenses nouvelles, énormes, que comporte l'électrification ; mais cela n'implique nullement que ce dernier mode de traction y doive être maintenu, bien au contraire, les moteurs Diesel étant susceptibles de fournir, pour ces petites exploitations, un nouveau système de traction aussi souple, pratique et économique (1) que particulièrement adapté aux besoins de ces lignes, pour lesquelles, avee l'interposition de la transmission électrique, ils semblent vraiment faits, quoique également applicables, $\mathrm{du}$ reste, aux exploitations de grandes lignes, toujours plus spécialement visées lorsqu'il s'agit d'électrification.

intérêt à adopter, comme ses voisines, la fréquence-standarđ de 50 périodes, qui suffirait'à tous ses besoins, mais nécessiterait cependant l'installation de stations de conversion nombreuses pour pouvoir subvenir aux besoins de la traction ferroviaire moncphasée à 15 périodes. Encore n'est-il pas certain du tout que cette solution soit la meilleure en ce qui concerne la traction ; eu égard à l'orientation que prennent aujourd'hui le développement de la machinerie électrique et son perfectionnement en vue d'économies de courant toujours plus grandes, ladoption des moteurs à courant continu, plus souples sous de plus hauts voltages aux trolleys, paraissant bien devoir être sensiblement plus économique que celle des monophasés actuellement en service.

(1) Voir, à ce sujet, notre étude sur "La Traction ferroviaire par locomotives Electro-Diesel", parue dans "La Vie Technique et Industrielle » (décembre 1922 à février 1923). 